Anjou. Maine. Poitou-Charente. Touraine

108-4 | 2001

La Chambre des comptes de Bretagne

\title{
La clientèle des ducs de Montmorency à la Chambre des comptes de Nantes de 1542 à 1632
}

\section{Antoine Pacault}

\section{(2) OpenEdition}

12 Journals

\section{Édition électronique}

URL : http://journals.openedition.org/abpo/1686

DOI : 10.4000/abpo. 1686

ISBN : 978-2-7535-1484-3

ISSN : 2108-6443

Éditeur

Presses universitaires de Rennes

Édition imprimée

Date de publication : 20 décembre 2001

Pagination : 171-193

ISBN : 978-2-86847-674-6

ISSN : $0399-0826$

\section{Référence électronique}

Antoine Pacault, «La clientèle des ducs de Montmorency à la Chambre des comptes de Nantes de 1542 à 1632 », Annales de Bretagne et des Pays de l'Ouest [En ligne], 108-4 | 2001, mis en ligne le 20 décembre 2003, consulté le 20 avril 2019. URL : http://journals.openedition.org/abpo/1686 ; DOI : $10.4000 / a b p o .1686$ 


\title{
La clientèle des ducs de Montmorency à la Chambre des comptes de Nantes de 1542 à 1632
}

\author{
Antoine PACAULT \\ Professeur certifié d'histoire et géographie \\ Lycée Jules-Verne de Rennes
}

Il n'était pas rare, au XVI ${ }^{\mathrm{e}}$ siècle et encore aux débuts du XVII ${ }^{\mathrm{e}}$, de rencontrer dans les cours royales bretonnes, ou en d'autres charges royales, aux plus hautes responsabilités comme aux plus petites, des officiers liés à de grands seigneurs de la province, soit qu'ils fussent en même temps employés à leur service, ou qu'ils l'aient été précédemment, eux ou leurs proches, ou qu'ils entrassent simplement en relations occasionnelles avec eux, leur rendant tel ou tel service ponctuel. On en trouvait à Rennes (les familles d'Argentré et Hay, par exemple, y étaient de la clientèle des comtes de Laval et barons de Vitré; Jean de Mésanger, conseiller au parlement de 1568 à 1586, était employé au service du marquis d'Élbeuf baron d'Ancenis, etc.). On en trouvait aussi à Nantes, à la Chambre des comptes. En étudiant les officiers de finances bretons de la première moitié du XVI ${ }^{\mathrm{e}}$ siècle, Dominique Le Page ${ }^{1}$ en a repéré vingt-cinq (dont onze membres de la Chambre des comptes) qu'il considère comme liés à de grands seigneurs. Ainsi par exemple François de Kermainguy, président à la Chambre des comptes en 1548 après son père, et qui était aussi sénéchal de Blain pour le vicomte de Rohan. Après 1550, des situations comparables existèrent aussi : Jehan Apvril, sieur de Lourmais, payeur des gages à la Chambre, puis trésorier des États pendant plus de quarante-cinq ans, était aussi trésorier et receveur général de François de Coligny, qui tenait Laval et Vitré pour son fils Guy-Paul (Guy XIX de Laval); et plus tard, Jean Blanchard de Lessongère, premier président à la Chambre des comptes de 1634 à 1641, provenait de la maison de César de Vendôme dont il avait été le superintendant de 1619 à 1630 . Ce ne sont là que des exemples parmi les plus frappants.

À chacune des rencontres de ce genre, on est amené à parler d'une clientèle des Grands à la Chambre des comptes. Mais dire cela n'explique

1. Dominique Le PAGE, Finances et politique en Bretagne au début des temps modernes. Paris, 1997 (thèse, Brest 1995). Voir $3^{\mathrm{e}}$ partie, p. 226 à 232. 
pas tout. Le double service que rendaient apparemment ces hommes, au roi et à un maître particulier, peut $a$ priori correspondre à plusieurs situations : le service du seigneur demeurait-il fondamental, celui du roi ne venant que le compléter, ou le récompenser? Au contraire, le service du roi n'était-il pas, ou ne devenait-il pas le seul but réellement recherché, celui du seigneur n'étant qu'un marchepied préliminaire ou un adjuvant de circonstance? Ces deux services étaient-ils rendus avec une égale conviction, ou le seigneur ne disposait-il à la Chambre que de bonnes volontés occasionnelles et prudentes? On se pose ces questions en devinant que tous les cas se rencontraient peut-être, mais en sentant aussi qu'on ne pourra pas y répondre complètement. On ne sait pas ce qu'il y avait dans les têtes et dans les cours. On peut tout au plus identifier quelques hommes et regarder certains de leurs comportements.

On peut s'attendre à trouver des évolutions dans ces comportements. Après la montée de l'influence des Grands en Bretagne sous François ${ }^{\text {er }}$ (laquelle venait naturellement prendre la place de celle de la reine Anne après 1514, ou de la reine Claude après 1524; et elle pouvait aussi correspondre au souhait du roi de faire mieux relayer son autorité) $)^{2}$, les périodes suivantes durent être marquées par d'autres inflexions. On peut chercher à préciser tout cela en observant de près ce que fut la clientèle des ducs de Montmorency à la Chambre des comptes entre 1542 et 1632, période où ils possédèrent en Bretagne la baronnie de Châteaubriant (cette partie de ses domaines que Jean de Laval avait donnée à sa mort au connétable Anne) ${ }^{3}$. Ils faisaient alors partie des plus importants seigneurs de la province, mais ils ne vinrent que très peu y séjourner (Anne de Montmorency le fit, avec le roi, en 1551 et 1565, et ce fut sans doute tout; quant à ses successeurs, ils vinrent encore moins souvent). Par contre, leur puissance dans l'État était généralement grande, plus que celle d'aucun des autres seigneurs de la province. Toutefois, elle varia au cours de ce siècle que nous allons envisager, et, au moins pour cette raison, il faut distinguer plusieurs périodes.

\section{Ibidem, p. 231.}

3. Le 5 janvier 1539, Jean de Laval, baron de Châteaubriant et gouverneur de Bretagne depuis 1531, fit donation du tiers de tous ses biens au connétable de Montmorency, sous réserve d'usufruit jusqu'à sa mort, qui survint en 1542. Montmorency vint prendre possession dès le 5 mai 1540 (Dom Morice, Preuves..., III, col. 1034). Les seigneuries que Montmorency acquit ainsi étaient étendues et jointives, dans le nord du comté nantais et le sud du diocèse de Rennes, et comprenaient, autour de la terre de Châteaubriant proprement dite, les baronnies ou châtellenies de Rougé, Derval, Vioreau, Nozay, Issé, Teillay, Jans et Guémené, Rieux-en-Nort, auxquelles s'ajoutait, en Anjou, toute proche, la baronnie de Candé. La même année, Claude de Villeblanche fit au connétable une donation semblable de ses seigneuries de Martigné-Ferchaud (diocèse de Rennes), et de Callac, Plusquellec et Plougonver (diocèses de Tréguier et de Quimper). Montmorency y ajouta encore, par achats, les seigneuries de Brancien et du Chastelier (diocèse de Saint-Malo) en 1540, sur Claude de Villeblanche, la châtellenie d'Oudon (diocèse de Nantes) en 1553, et celle de Champtoceaux (en Anjou) en 1558, sur le duc d'Étampes, ainsi que quelques petites seigneuries mêlées à ses autres terres. L'ensemble n'équivalait pas à ce qu'avait possédé Jean de Laval, mais était quand même considérable. 
Malgré quelques moments de disgrâce (en 1541-1547, puis en 1559), le connétable Anne de Montmorency (devenu duc de Montmorency en 1551) fut à peu près constamment un personnage considérable. Il avait la confiance du roi, comme le montrent les charges qu'il exerçait, et sa très longue présence aux affaires. Il en était si proche que son appui, qu'il savait exercer, était le plus efficace et le plus recherché, et que, pour beaucoup, servir le roi et servir le connétable en même temps allaient de soi, comme deux tâches complémentaires. Par ailleurs, Jean de Laval, dont il se trouvait l'héritier, avait été lui-même un personnage puissant (par l'étendue de ses domaines, par la faveur royale, par sa charge de gouverneur de la province qui en témoignait, et aussi par son amitié avec Montmorency), et pouvait avoir disposé d'une clientèle solide, qui allait naturellement chercher à se reporter sur son successeur.

On ne s'étonne donc pas de voir qu'au milieu du Xvi ${ }^{\mathrm{e}}$ siècle, et pendant vingt à trente ans, le connétable de Montmorency, nouveau possesseur de la baronnie de Châteaubriant, eut recours aux officiers des finances nantais pour exercer la responsabilité d'ensemble de ses domaines bretons. Nous voyons ainsi que ce fut le président aux comptes Gilles de la Pommeraye $^{4}$ qui le conseilla lors de la donation à lui faite par Jean de Laval, en 1539, et qui fut chargé de la prise de possession des terres (et sans doute ensuite d'en superviser l'administration), en même temps qu'il le conseillait aussi sur tout autre sujet, comme la tutelle de la jeune Charlotte de Laval (fille de Gui XVI de Laval), et qu'il s'entremettait sur ce point avec le seigneur de Châteaubriant. Ce fut lui qui eut la procuration du connétable pour conclure en 1540 l'achat des terres de Brancien et du Chastellier, dont il arrondissait le don que lui avait fait de ses domaines Claude de Villeblanche. Montmorency avait toute confiance en lui : "Vous avez en cella besongné en homme qui entend les matières, et n'eussiez pu mieux faire $^{5}$ ", lui écrivait-il à propos des difficultés nées de la donation de Claude de Villeblanche.

Mais Gilles de la Pommeraye était de la clientèle du connétable, et membre de son conseil, bien avant son entrée à la Chambre des comptes en 1537, laquelle s'expliquait sans doute justement par sa protection ${ }^{6}$. Dès 1535, il était le procureur de Montmorency, devant le sénéchal de Rennes,

4. Gilles de la Pommeraie, sieur de la Pommeraie (en Caro) et d'Entrammes (au Maine), fut premier président à la Chambre des comptes du 18 avril 1537 au 27 mars 1545 . Il avait été échanson, puis panetier et maître d'hôtel du roi, et avait mené plusieurs ambassades, notamment en Angleterre en 1531, 1532, 1533, 1536, 1543 (Dominique LE PAGE, op. cit., p. 632-633). Il épousa Jeanne Le Jeune, dame de la Morlaye (en Missiriac), où il demeurait en 1540. Les terres de Brancien et du Chastelier (en Éréac et Lanrelas) furent vendues par Claude de Villeblanche à Montmorency pour compléter la donation qu'il venait de lui faire de Martigné et de Callac.

5. Bertrand de Broussillon, La Maison de Laval, 1020-1605. Cartulaire de Vitré. Paris, 1902, t. 4. p. 153, lettre de Montmorency à La Pommeraye du 4 avril 1540.

6. Ce qu'affirme Dominique Le Page (op. cit.), qui pense même que ce fut Montmorency qui versa les 12000 livres du prix de son office, selon une pratique dont bien d'autres exemples existent au XvI ${ }^{\mathrm{e}}$ siècle. 
lorsqu'il fallait décider de faire de René de Montjean le curateur du jeune Gui XVII de Laval, au moment de son mariage avec Claude de Foix ${ }^{7}$, et il le représenta à ce mariage, qui eut lieu à Châteaubriant les 22 et 23 octobre 1535. Dès avant 1531, même, il fréquentait le connétable, puisque nous découvrons que Gui XVI de Laval profitait d'une visite qu'il partait lui faire pour le charger d'une lettre ${ }^{8}$. Pas plus que le président de Cucé (Julien Bourgneuf, sieur de Cucé et d'Orgères, président aux Grands Jours de Bretagne), déjà au service de Montmorency en 1540 au moins ${ }^{9}$, Gilles de la Pommeraye n'était donc une personnalité bretonne que le connétable aurait eu besoin de recruter pour s'occuper de ses domaines de Châteaubriant, lorsqu'il en devint le possesseur. Il était entré à son service bien avant 1542, et bien avant l'acquisition de son office nantais. Surtout, il était à l'origine un officier du roi, et il ne cessa pas de l'être. C'était par ses charges et commissions royales (maître d'hôtel, ambassadeur) qu'il avait (sans doute) eu l'occasion d'être connu de Montmorency et de le servir, bien avant 1539 ou 1542. Il avait deux maîtres. Pendant quinze à vingt ans au moins, il fut constamment, auprès du connétable ou en Bretagne, à la fois un serviteur du roi et un serviteur de Montmorency, les deux services s'appuyant naturellement et se confondant.

Probablement peut-on dire la même chose de Florimond Le Charron. En 1552, Montmorency donna à Florimond Le Charron, sieur de la Pabotière, trésorier et receveur général pour le roi en Bretagne depuis 1540, une procuration générale pour " comparoir pour nous, nos causes défendre, notre personne représenter en jugement et dehors par devant tous juges, commissaires et autres ${ }^{10} \ldots$..., ce qui ne faisait d'ailleurs que le confirmer en des responsabilités qu'il exerçait déjà en fait, au moins dès 1551. C'était lui qui surveillait et contrôlait la marche des procès qu'avait alors

7. Bertrand de Broussillon, op. cit., t. 4, lettre de Montmorency à la Pommeraye du 22 octobre 1535. Le contrat de mariage fut établi au château de Châteaubriant, devant Pierre d'Argentré, sénéchal de Rennes, le 22 octobre, et le mariage eut lieu dans la chapelle du château le 23 (Arch. dép. d'Ille-et-Vilaine, $1 \mathrm{~F} 44$ ).

8. Ibidem, t. 4, lettre s.d. de Gui XVI à Montmorency (entre 1525 et 1531).

9. Parce que, par sa belle-famille, il appartenait au milieu parlementaire parisien, ibid., t. 4. p. 153, lettre de Montmorency à La Pommeraye du 4 avril 1540. Le connétable réunissait alors son conseil, où figurait le président de Cucé, pour parler des difficultés que suscitait le sieur de Broons, lequel contestait la donation que venait de faire au connétable Claude de Villeblanche. Julien Bourgneuf, sieur de Cucé et d'Orgères, né vers 1500, fils de noble homme Jean Bourgneuf, prévôt des monnaies à Rennes, anobli par la reine Anne en 1506, et de Jeanne Thierry, épousa à Paris en 1525 Anne Dauvet, fille de Guillaume Dauvet, seigneur de Clagny, conseiller au parlement de Paris (et petite fille de Jean Dauvet, procureur général en 1446, et premier président en 1466).

10. Bibli. nat., ms. fr. 18 633, 20 janvier 1552. Les trésoriers de France en Bretagne n'étaient pas membres de la Chambre des comptes. Ils avaient toutefois le droit d'y entrer. Florimond Le Charron, sieur de la Pabotière, avait été commis au paiement des officiers domestiques du roi en 1535, était devenu commis à la trésorerie de Bretagne à partir de décembre 1538, et trésorier en 1540. Avant d'être institué receveur général de la baronnie de Châteaubriant pour Montmorency, on le signale comme " gérant le fait " de Jean de Bretagne, comte de Penthièvre (Dominique LE PAGE, op. cit., p. 415). 
le connétable contre tous les autres héritiers de Jean de Laval, et qui l'en informait régulièrement. En même temps, il était le receveur général de Châteaubriant. On le trouve même cité dans une lettre de Montmorency dès 1540. Son maître devait bien le connaître, pour lui laisser une autorité aussi étendue, et c'est vraisemblable, car Florimond Le Charron, comme La Pommeraye, avait été employé auprès du roi avant sa venue en Bretagne en 1538, et pourvu notamment, en 1533, de l'office de receveur et payeur des gages des officiers domestiques du roi. Comme la Pommeraye aussi, il conserva jusqu'à sa mort ses responsabilités importantes en Bretagne au service de Montmorency comme à celui du roi; et, comme La Pommeraye encore, il ne pouvait cumuler ces deux services qu'avec l'accord du roi, et parce que le roi y trouvait lui aussi son intérêt : alors qu'en 1511 la reine Anne avait répété (après ses prédécesseurs) qu'aucun de ses officiers ne devait « tenir autre office que d'elle ${ }^{11}$ ", François $I^{\mathrm{er}}$ a bien pu, délibérément, chercher à appuyer par de tels cumuls l'autorité des Grands dans la province (de Montmorency comme des autres) pour mieux, par eux, y asseoir la sienne ${ }^{12}$. Le souvenir du trésorier Charron, pendant plusieurs années après sa mort (début 1560), demeura en tout cas comme une référence que citaient volontiers en leurs lettres les serviteurs du duc.

La Pommeraye et le trésorier Charron n'avaient d'ailleurs certainement pas été seuls, en ces années, à veiller depuis Nantes aux affaires de Châteaubriant : il y avait aussi Jean d'Alesso, sieur de Lezeau, maître des comptes de Nantes de 1536 à 1552, qui était un serviteur de Montmorency, qui avait sans doute lui aussi dû à cela son office nantais, mais dont l'arrivée à la Chambre des comptes en 1536, comme celle de La Pommeraye l'année suivante, marquait aussi peut-être le désir du roi de mieux faire sentir et appliquer ses volontés dans la province, mieux qu'il ne pouvait le faire par des officiers au recrutement étroitement provincial ${ }^{13}$. Il aidait à l'administration des domaines bretons de son maître après 1542, allant par exemple, en 1551, visiter les bois et forêts de Châteaubriant et Villocher ${ }^{14}$,

11. Arch. dép. d'Ille-et-Vilaine, 1 F 44-46 (fonds Hévin). La reine Anne obligea ainsi en octobre 1511 son procureur de Rennes, Yves Bruslon, à quitter la charge de sénéchal de la vicomté de Rennes qu'il exerçait en même temps pour le comte de Laval.

12. Dominique LE PAGE, " Noblesse et pouvoir royal en Bretagne (1480-1540) ", dans Noblesses de Bretagne du Moyen Âge à nos jours, sous la direction de Jean KerHERVE, Rennes, 1999.

13. Dominique Le PAGE, "Jean de Plédran (1535-1540) ", Études sur la Bretagne et les pays celtiques, Brest, 1994, p. 119-139.

14. Dominique LE PAGE, Finances et politique..., op. cit., p. 625-626. Jean d'Alesso, sieur de Lezeau, fut maître des comptes à Nantes en 1536 (et résigna sa charge en 1552), fut pourvu maître des comptes à Blois le 9 mars 1546, et à la Chambre des comptes de Paris en 1554. Il mourut à 59 ans le 13 septembre 1572. Il était à l'origine un serviteur de Montmorency, et il le resta toute sa vie. Même après son départ de Bretagne, il continua de surveiller les affaires de la baronnie de Châteaubriant, par ses fonctions d'intendant général du connétable, et à s'y rendre à plusieurs reprises. Il pouvait profiter, pour le faire, de ce que des commissions royales l'amenaient à se rendre dans la province : en novembre 1555, il fut à Guingamp pour procéder à « la réunion et réformation de la comté de Penthièvre et seigneurie de Guingamp " (Dom Morice, Preuves..., III, col. 1151). 
ou inspecter Callac cette même année ${ }^{15}$. Lorsqu'après son départ de Bretagne d'Alesso fut maître des comptes de Blois, puis de Paris, il cumulait ces charges avec celle d'intendant général de Montmorency, puis de sa veuve, jusqu'à sa mort en 1572 .

Ces trois hommes - la Pommeraye, Florimond Le Charron, d'Alesso se ressemblaient en ce qu'ils inspiraient toute confiance au duc. Nous ne pouvons l'affirmer avec autant de certitude pour le receveur général de la baronnie de Châteaubriant qui vint ensuite. Pendant dix ans, de 1560 à 1569, nous voyons en ces fonctions Jacques Thévin, sieur de la Marsaulaye et de la Chotardière, autre receveur général des finances du roi en Bretagne. Il s'agissait là d'un Angevin (il avait été lieutenant des Eaux, Bois et Forêts d'Angers), et, s'il reprit le service de Montmorency qu'assurait son prédécesseur, et si on le choisit pour le reprendre, cela n'impliquait pas forcément de sa part une grande familiarité avec le duc, d'autant plus qu'on ne lui donna que la recette générale, et non pas (comme à Le Charron) une procuration pour s'occuper de toutes les affaires bretonnes. Mais si ce n'était peut-être pas un familier ancien du duc, du moins savait-il bien qu'en le servant il pourrait trouver un appui utile en l'exercice de sa charge royale. Et il savait le solliciter à l'occasion, ainsi en février 1562 où, après diverses nouvelles de sa recette de Châteaubriant, il demandait à Montmorency d'intervenir auprès de "Monsieur de Gonnort et autres messieurs les intendants de finances qu'ils ne souffrent que je sois chargé de payer au roi plus que mon état ne porte, comme l'on a fait l'année passée, dont je suis en peine comme vous l'avais dernièrement écrit pour avoir été chargé de XVm livres plus que ne pouvait porter ma charge ${ }^{16} \ldots$ " Aux côtés de Thévin, nous voyons agir quelques autres responsables, comme M. Charbonnier, un protestant de Saumur qui était par ailleurs fermier d'Oudon et de Champtoceaux, et qui ne devait guère être connu non plus à Paris. Mais enfin, Thévin était bien, comme ses prédécesseurs à la recette générale de Châteaubriant, un personnage important à Nantes, et bien placé pour remplir les charges que lui avait explicitement données le duc.

À côté de ces serviteurs manifestes du connétable, on peut sans grand risque supposer qu'il disposait naturellement d'autres sympathies et appuis possibles à la Chambre des comptes. Parmi eux, il y avait probablement les officiers des comptes issus du service de la baronnie, eux ou leurs auteurs : ne mentionnons pas Pierre Piraud (secrétaire de Jean de Laval, puis maître

En juin 1571, il fut commissaire du roi aux États de Bretagne qui se tenaient à Rennes. Il fit chaque fois en partant le détour par Châteaubriant (de LA LANDE DE CALAN, Documents relatifs aux États de Bretagne). Son fils, François d'Alesso, seigneur d'Éragny, maître des comptes de Paris de 1570 à 1594 (et mort en 1618) fut aussi au service de la connétable (au moins en 1581). Sa fille Anne, elle, épousa en 1559 Olivier Lefèvre d'Ormesson.

15. Arch. dép. des Côtes d'Armor, $19 \mathrm{~J}$ 22, où un compte du receveur de Callac le signale.

16. Musée Condé, série L, t. XVIII, $\mathrm{f}^{\circ} 145$, lettre de Thévin au duc de Montmorency du 15 février 1562. 
des comptes en 1540), qui mourut dès avant $1542^{17}$, mais Jehan Douette (auditeur des comptes en 1545) ${ }^{18}$, ou François Durand (auditeur des comptes de 1553 à 1557$)^{19}$ dont les familles avaient servi Jean de Laval et devaient naturellement se sentir attachées à Montmorency après lui, tant ces deux hommes avaient été familiers l'un de l'autre avant 1542. Et René Bonnier (maître des comptes, 1554) était certainement le frère de Jean Bonnier de la Gaudinais, procureur général de la baronnie au milieu du siècle, et donc l'oncle de Mathurin Bonnier de la Coquerie, son procureur fiscal jusqu'à la Ligue $^{20}$. À notre connaissance, aucun de ces hommes ne reçut toutefois de commission particulière pour s'occuper des affaires de Châteaubriant.

Les serviteurs des comtes de Laval, eux aussi, étaient sans doute attentifs aux intérêts du connétable à la Chambre des comptes (comme ils le furent à Rennes vers 1545 lors du procès qu'il y eut contre la dame d'Acigné), tant leurs maîtres étaient liés : " Je ne scay en ce monde chose qui me vienne à plus grant plaisir que d'avoir l'heur de vous povoir faire service et vous obéyr ce que je ferai toute ma vie ", écrivait Gui XVII à Montmorency le 25 juin 1545, à propos de ce procès ${ }^{21}$. Jean de Laval avait été très proche de

17. Pierre Piraud avait été secrétaire de Jean de Laval, puis maître des comptes du 6 octobre 1540 jusqu'à sa mort, avant le 19 mars 1541. Il avait épousé Perrine Vivien, fille de Jean Vivien, secrétaire du comte de Laval en 1510 (Dominique LE PAGE, op. cit.), ou de P. Vivien, receveur général de Jean de Laval en 1509-1510. Il avait acquis la terre de la Touche, en Nozay (de Bernard Tregouët, receveur de l'évêché de Rennes), ainsi que cinq autres terres dans la même paroisse, qui toutes allaient revenir à Claude de Commaille (fille du second mariage de Perrine Vivien), et, par elle, aux Cornulier.

18. Noble Maître Jehan Douette, sieur de la Grant Noë, clerc et secrétaire de la Chambre des comptes de 1545 à 1557 (Dominique LE PAGE, op. cit., p. 609), par résignation de Gilles Bricaud son beau-père. Il était le fils de Jehan Douecte et de Perrine Théaudin, et il épousa Françoise Girault. En 1524, Pierre Douette, ou Douecte, était argentier de Jean de Laval (Arch. dép. d’Ille-et-Vilaine, 2 Er 204), tandis que Jehan Douecte, sieur de la Grant Noë et père de l'auditeur des comptes, figurait parmi les vassaux de Châteaubriant (sous Nozay) (Bibli. nat., ms. fr. 22331), et parmi ceux de Marsac (Arch. dép. d'Ille-et-Vilaine, 3 H 129). Il y a peu de risques à penser qu'il s'agissait bien de la même famille.

19. François Durand, sieur du Pontpiétain (en Blain), succéda le 2 janvier 1553 à Jean de la Rivière comme greffier de la Chambre des comptes. Il l'était encore en 1557 (Dominique LE PAGE, op. cit., p. 648). Il n'était pas ce Jehan Durand, sieur de la Minière (en Rougé), qui avait été serviteur de Jean de Laval, au moins vers 1520, mais il devait être de la famille. Au milieu du siècle, le seigneur de la Minière était un autres François Durand (fils de Jehan).

20. René Bonnier, appelé sieur de la Gaudinais (en Rougé) dans les registres paroissiaux de Saint-Jean de Béré en 1545, fut admis comme maître des comptes en 1554, et est mort au cours des années 1560. Il était issu d'une famille de fermiers (et juges) de la baronnie de Châteaubriant, qui demeurait à Rougé, et y était probablement venue de MartignéFerchaud aux débuts du siècle. Par l'étendue de ses biens, elle l'emportait de beaucoup sur toutes les autres familles notables de la région. De René Bonnier lui-même, nous ne savons pas grand chose. Qu'il ait été le frère de Jehan Bonnier, sieur de la Gaudinais, le procureur général de Châteaubriant en ces années-là, n'est qu'une forte probabilité.

21. Bertrand de Broussillon, op. cit., t. 4, p. 218-219, lettre de Gui XVII à Montmorency du 25 juin 1545. La dame d'Acigné (Anne de Montejean, veuve de Jean V d'Acigné) contestait au connétable la donation que lui avait faite Jean de Laval, et réclamait pour elle les terres que Jean de Laval avait recueillies de Françoise de Rieux sa mère (Derval, Fougeray, Châteaugiron...). Le sénéchal de Rennes, Pierre d'Argentré, était un serviteur de la maison de Laval. 
son cousin, au service de qui passèrent naturellement, à sa mort, certains de ses serviteurs ou de ses gentilshommes ${ }^{22}$. Et Montmorency lui-même l'était tout autant, ou plus encore : il était le beau-frère de Gui XVI (mort en 1531), et donc l'oncle (et le tuteur, conjointement avec Jean de Laval) du jeune Gui XVII. Pendant plusieurs années, les intérêts du connétable en Bretagne ne furent donc pas seulement ceux des domaines qu'il venait d'hériter, mais presque autant ceux des vastes possessions de son neveu : Laval, Vitré, Montfort, Quintin, etc. Il s'en préoccupa, nous en avons des preuves ${ }^{23}$, et on peut vérifier que ni la majorité de Gui XVII, ni peut-être même sa mort (en 1547), qui fit lui succéder sa nièce Renée de Rieux (Guyonne XVIII), puis les Coligny (qui étaient aussi par ailleurs de ses neveux) suffirent à le détourner de s'intéresser aux seigneurs de Laval et Vitré.

Parmi les serviteurs de la maison de Laval à la Chambre des comptes, qui ne pouvaient que rentrer dans la clientèle du connétable, nous voyons surtout Antoine Dessefort (auditeur des comptes de 1529 à 1545), et son fils Michel (qui reprit la charge de 1545 à 1555). Le premier avait été, au moins vers 1523, trésorier et receveur de Gui XVI, au service duquel on avait aussi rencontré Maître Alexandre Dessefort, ancien secrétaire de la reine Claude de France, avant 1524, et qui cherchait en 1530 à rentrer dans la maison de la nouvelle reine grâce à Montmorency qui, comme Grand Maître, était alors chargé de constituer ${ }^{24}$.

22. Les mêmes serviteurs, ou leurs familles, fréquentaient concurremment le service des deux seigneurs. Pierre Le Forestier, seigneur de Kerahuys et de la Touche-Berthelot, époux de Bertrande de la Bouhère, et mort en 1541 environ (et dont Dubuisson-Aubenay vit en 1636 la tombe chez les Cordeliers de Saint-Brieuc), était en 1514-1516 maître d'hôtel de Jean de Laval, et il fut ensuite sénéchal de Quintin, pour le comte de Laval (en 1532), et peut-être aussi alloué de Ploermel (en 1534). Son fils, autre Pierre Le Forestier, fut greffier aux Grands Jours à partir de 1533. On voit aussi que P. Vivien était argentier de Jean de Laval aux débuts du siècle, cependant que Jean Vivien était secrétaire de Gui XV de Laval, et que Jacques Vivien fut procureur de Châtillon pour Gui XVI, en 1527, puis lieutenant de Vitré. François Legouz, sieur de Gallerne, était un gentilhomme angevin qui fut successivement (ou simultanément) attaché à Jean de Laval, à Gui de Laval et à Montmorency.

23. Ce fut Montmorency qui obtint en 1543 que le comté de Montfort ne relevât plus de la justice royale de Ploermel, mais directement de celle de Rennes. Un écrit du début du XVII ${ }^{\mathrm{e}}$ siècle (Arch. dép. d'Ille-et-Vilaine, $1 \mathrm{~F}$ 745), rappelant ce fait, lui en attribuait bien l'origine, comme tuteur de Gui XVII, mais le disait aussi « lors gouverneur de ce pays et duché de Bretagne ", erreur intéressante à relever. On peut remarquer aussi que ce ne fut qu'après la mort de Gui XVII que les créanciers de la maison de Laval purent obtenir la mise en vente de plusieurs de ses terres.

24. Bertrand de Broussillon, op. cit., t. 4, p. 100, lettre de Gui XVII à Montmorency du 11 juillet 1530. Alexandre Dessefort fut trésorier de l'Épargne du duché (Dominique LE PAGE, op. cit., p. 426). On voit comment le maître, Gui XVI, après avoir recueilli un serviteur de la reine privé de son emploi, et l'avoir bien apprécié (il le disait), cherchait à le pousser en le recommandant à Montmorency. Antoine Dessefort, sieur du Breil (en Bouguenais) : d'une famille originaire du Berry, où il était né en 1480, il devint auditeur des comptes par le décès de Pierre Honoré de la Forest, et fut reçu en 1529, après avoir été receveur de Nantes de 1516 à 1528, et receveur du fouage de Vannes de 1520 à 1528 (Dominique LE PAGE, op. cit., p. 606-607), et aussi trésorier général de Gui XVI de Laval 
Ainsi, même si, dans ce qui précède, il n'y a pas uniquement des certitudes, mais aussi plusieurs suppositions simplement vraisemblables, on peut quand même conclure qu'Anne de Montmorency avait des relations solides et efficaces parmi les officiers de finances nantais.

En 1571, après la mort du trésorier Thévin, ce fut encore dans le milieu des officiers de finances nantais qu'on chercha à lui trouver un remplaçant pour Châteaubriant, avec une responsabilité toujours limitée aux seules affaires financières de la seigneurie. Mais les temps avaient changé : le connétable avait été tué, en 1567, au combat de Saint-Denis, et c'était depuis lors sa veuve, Madeleine de Savoie, qui possédait la baronnie de Châteaubriant (jusqu'à sa mort en 1586). Il n'était pas question de trouver un homme déjà connu et d'une fidélité éprouvée, car il n'y en avait pas, ou plus. Il s'agissait seulement de choisir parmi les candidats qui se manifestaient, et que guidait seulement sans doute l'intérêt que le service de Montmorency aurait pour leur carrière. On peut juger que cet intérêt leur apparaissait encore grand, puisque trois personnages " capables de faire ladite charge " se mirent sur les rangs, et que deux d'entre eux proposaient même de l'exercer gratuitement (le troisième demandant seulement 300 livres par an). Mais on remarque qu'aucun des trois n'avait d'office royal aussi important que les précédents, et qu'ils provenaient tous du milieu le plus strictement local.

L'un était Guillaume Meneust, sieur de la Rouaudière. C'était un auditeur des comptes, pourvu en 1570, après avoir été depuis 1548 garde des livres à la Chambre. Il était en même temps greffier des États de Bretagne, dont il avait été l'un des députés vers le roi à Moulins en 1565. Il était donc bien au service du roi. Mais, parallèlement, il devait déjà appartenir de quelque façon à la clientèle de Montmorency. Nous le devinons en voyant que le duc l'avait appelé auprès de lui à Châteaubriant en 1565, alors qu'il y séjournait quelques semaines avec le roi. Que Guillaume Meneust fût allé demander lui-même à Paris la charge de receveur de Châteaubriant montre aussi qu'il ne devait pas y être totalement inconnu : "Monsieur Le Meneust, dernièrement à Paris, qui a parlé à vous, à monsieur de Vincelles et à moy, a fait offre de faire ladite recette sans aucuns frais, et que seulement vous ferait compter le prix du port et voiture des deniers qui seraient mis entre vos mains, sans prendre aucuns gages ${ }^{25} \ldots$ ". Il mêlait donc sans doute les deux services, comme l'avait fait son père Guy Meneust, qui avait été avant 1548 garde des livres à la Chambre des comptes de Bretagne, et

(vers 1523 ou avant). Son fils Michel Dessefort fut auditeur des comptes de 1545 à 1555 , et en même temps avocat général (1554-1556), puis conseiller au parlement de Rennes en 1556. Il mourut avant 1568.

25. Musée Condé, série L, t. XXI, f ${ }^{\circ}$ 141, 26 avril 1571, lettre d'Alesso de Lezeau à la duchesse de Montmorency. Monsieur de Vincelles, cité par cette lettre, était Toussaint Barrin, qui présidait le conseil du duc, et dont le neveu Jacques Barrin allait être l'année suivante envoyé en Bretagne. 
en même temps auditeur ou maître à celle du comte de Laval aux débuts du siècle ${ }^{26}$. Le désir de Guillaume Meneust de devenir le serviteur en titre de Montmorency prolongeait une habitude familiale ancienne, qui existait aussi dans la famille de sa femme Bertranne Godet (au service elle aussi des comtes de Laval, et de Montmorency) ${ }^{27}$, et qui se poursuivit chez Guy Meneust, le fils de Guillaume, qui fut pourvu en mars 1570 de la charge de procureur général de la reine mère au parlement de Bretagne et en ses baronnies de Fougères, Bazouges, Rhuis, Antrain et Saint-Aubin du Cormier $^{28}$. On retrouvait d'ailleurs la même ambition de servir les Grands chez Gilles Meneust, sieur de la Vieuville, frère de notre Guillaume, qui fut jusqu'à sa mort en 1587 receveur de la baronnie de Fougères (à la reine mère). Avec Guillaume Meneust, nous devinons donc une famille qui est passée naturellement des charges financières seigneuriales aux charges royales, mais qui continuait à chercher le protecteur le mieux capable de soutenir sa réussite. Après les comtes de Laval, ses maîtres initiaux, elle se tournait donc vers 1570 à la fois vers Montmorency (avec Guillaume), et vers Catherine de Médicis, propriétaire de la baronnie de Fougères (avec Guy, avec Gilles son oncle, et avec divers autres cousins aussi).

Le second candidat à la recette de Châteaubriant, François Trottereau, sieur du Palierne (en Moisdon), n'était pas encore le trésorier et receveur général des Finances qu'il devint en 1572, ni le maître des comptes qu'il allait aussi devenir, en 1577. Il était toutefois déjà engagé lui aussi au service du roi dans la province, comme receveur alternatif des fouages de Tréguier (pourvu en 1568). Mais il provenait au départ du service du baron de Châteaubriant, puisqu'il en avait été le châtelain et fermier pour plusieurs de ses terres, au cours des années 1560, et qu'il l'était encore en 1570. Cette année-là, il fit même fonction de receveur de toute la baronnie,

26. À coup sûr en 1543-1544, et probablement dès 1519, Guy Meneust était auditeur (ou maître) à la Chambre des comptes de Laval (Arch. dép. d'Ille-et-Vilaine, 1 F 1537 et 1540). Cette famille Meneust avait grandi au service des comtes de Laval, en leur baronnie de Vitré qui était sa région d'origine.

27. Julien Godet, procureur de Montmorency au parlement de Bretagne depuis 1559, venait alors d'être nommé lieutenant de Châteaubriant (entre 1567 et 1570), et d'être remplacé par son frère Guillaume en sa charge rennaise. Tous deux descendaient d'anciens serviteurs des seigneurs de Châteaubriant et de Laval en leurs terres du Theil, de Tinténiac (voir infra la note 36).

28. Arch. dép. d'Ille-et-Vilaine, série 1 BA. Maître Guillaume Meneust, auditeur des comptes, et Guy Meneust son fils, conseiller et procureur de la reine mère en Bretagne, furent tous deux anoblis par lettres du 29 décembre 1577 (Arch. dép. d'Ille-et-Vilaine, 1 BA/7, f ${ }^{\circ}$ $304 \mathrm{v}$ et $306 \mathrm{r}$ ). À cette famille appartenaient certainement aussi (des cousins ?) Jean Meneust, qui fut receveur ordinaire du domaine royal de Fougères de 1547 à 1569 (Dominique LE PAGE, op. cit.), et cet autre Jean Meneust qui était sénéchal de Fougères à la veille de la Ligue, et qui fut décapité à Rennes en août 1589 pour y avoir apporté la nouvelle de la mort du roi. À Gilles Meneust, frère de Guillaume, qui fut receveur de la baronnie de Fougères jusqu'à sa mort en 1587 avait aussi succédé un (autre?) Jean Meneust, receveur du domaine de Fougères signalé dans l'état des finances de Bretagne d'avril 1589 à septembre 1591 (Gaston de CARNE, Documents sur la Ligue en Bretagne, 2 vol., Nantes, 1889). 
exerçant de fait cette charge de recette générale dont il voulait être pourvu en titre : "Il est celui qui a fait la recepte depuis la mort dudit Thévin, et m'a dit qu'il vacquera tant à la recepte de vos deniers qu'à vos autres affaires... C'est un homme fort diligent ${ }^{29}$. " Comme l'avait fait la famille Meneust, François Trottereau était en train de passer de la recette seigneuriale aux offices financiers royaux; et comme dans la famille Meneust, François Trottereau devait savoir de quel intérêt le service et l'appui d'un Grand pouvaient être pour une carrière. On devine une ambition en action. Mais, en 1571, on n'en était encore qu'aux débuts de l'ascension. L'essentiel était à venir, y compris l'entrée dans le monde des notables nantais que lui permit son mariage avec Jeanne Harouys. Pour l'instant, il n'était encore qu'un serviteur local de Montmorency, et il n'en était guère connu : on n'en parlait à Paris que comme d'" un nommé Trotereau ". Il n'avait sans doute pas encore de domicile à Nantes, et il demeurait vers Châteaubriant, en sa terre du Palierne (en Moisdon), achetée dix à douze ans plus tôt, ou en sa maison dite du Palierne, qu'il avait acquise peu après dans la ville ${ }^{30}$.

Le troisième candidat, qui fut brièvement choisi, Antoine Charton (ou Charreton), sieur de Monteilles (Montaigle), maître des comptes en 1568, avait pour lui d'avoir été le commis (et le beau-frère) du trésorier Thévin, avec le fils duquel il avait rédigé le dernier compte. Ses qualités étaient soulignées : "Il est abille homme et de bon esprit, et a autorité dans le pays... je le dis sans affection ${ }^{31}$... " Ce fut peut-être sa familiarité connue avec la tâche à attribuer qui le fit finalement désigner.

Peut-on considérer que ces trois hommes étaient des clients de Montmorency à la Chambre des comptes? Le terme serait sans doute trop fort. Aucun ne peut être évidemment comparé à La Pommeraye, d'Alesso ou Le Charron. Ils ne tenaient pas d'aussi près à la maison du duc. Il y avait

29. Musée Condé, série L, t. XXI, f ${ }^{\circ} 141,26$ avril 1571.

30. François Trottereau fut institué receveur alternatif des fouages de Tréguier le 8 octobre 1568, et remplacé en sa charge le 3 août 1572 par Ymbert de Launay. En 1572, il fut fermier des devoirs établis dans l'évêché de Nantes pour le rachat du domaine. Il devint trésorier et receveur général des finances à la résignation de François Miron (le 20 mars 1572), et il résigna cet office le 21 septembre 1574. Il fut pourvu d'une charge de maître des comptes le 26 janvier 1577 (office de la création de 1575). François Trottereau était probablement d'origine tourangelle (et frère de Jean Trottereau, chanoine de Saint-Malo, official et archidiacre de Porhoët). Il épousa Jeanne Harouys, ce qui fit de lui le beau-frère de Charles Harouys, sieur de la Rivière, conseiller au parlement, puis président au présidial de Nantes, et maire de Nantes en 1588; le beau-frère aussi de Louis Braillon de Barigny, d'Hélie Poyet, conseiller au parlement, puis de Georges Morin, sieur du Chapeau, maître des comptes en 1582 : il s'allia donc aux familles les plus notables de Nantes dans les années 1570-1580. François Trottereau avait acquis sa terre du Palierne, en Moisdon, en 1558-1559, et sa maison à Châteaubriant peu après. Il fut député aux États de Bretagne en 1567 et en 1570 . Il mourut en 1588.

31. Musée Condé, série L, t. XXI, $\mathrm{f}^{\circ}$ 141, 26 avril 1571. Antoine Charton, maître des comptes en 1568, était comme Thévin un Angevin. En décembre 1569, il était allé en BasseBretagne pour aliéner le domaine du roi, et il en avait profité pour inspecter au passage la terre de Callac, qui était à la connétable de Montmorency. De retour à Nantes, en février 1570, il lui avait écrit pour lui rendre compte de cette inspection. 
certainement des liens qui avaient été noués avec Guillaume Meneust, mais ils furent insuffisants pour le faire choisir par la duchesse (Madeleine de Savoie). Et François Trottereau, pourtant fermier de la baronnie, était inconnu à Paris. Le désir de ces trois hommes de faciliter leurs carrières en se chargeant des affaires de Montmorency apparaît plus évident que la profondeur et l'importance de leur engagement antérieur dans ce service, quand il y en avait eu. Par ailleurs, aucun de ces trois hommes n'était d'un grand poids dans la province, pas plus qu'à la Chambre des comptes. Leur choix, pour cela, ne pouvait être très satisfaisant. Ce n'était qu'un pis-aller. C'est qu'il y avait à l'époque bien des affaires importantes à régler : il y avait, depuis la mort du connétable en 1567, la question du rachat dû pour ses domaines bretons, que la Chambre des comptes exigeait malgré le don que le roi en avait fait. En 1568, le procureur général de la Chambre avait seulement accepté de transiger à 500 livres pour chacune des terres, mais les fermiers de certaines de ces terres étaient jetés en prison pour défaut de paiement. Il y avait surtout les affaires pendantes en justice au parlement, à propos desquelles un simple officier des comptes était mal armé pour intervenir. Il fallait un vrai responsable des affaires bretonnes de Montmorency, et non pas seulement un simple comptable un peu arriviste :

" Il serait bien requis que vous eussyez par deçà un personnage qui sceust conduyre une infinité de procès que vous y avez et esquels vous estes poursuivie, et d'autres qui demeurent par faute de poursuite où vous avez grand dommage et perte, et aussi pour la réformation des rolles rentiers de vos terres et seigneuries avant que faire les baux de vos fermiers, car en ce faisant elles pourront augmenter ", écrivait à la duchesse un de ses envoyés en Bretagne, Myrevault, en février $1569^{32}$.

Cette demande était toujours sans vraie réponse deux ans plus tard. Elle montre que Montmorency ne pouvait plus trouver en Bretagne de client solide à qui confier ses affaires de Châteaubriant, et sans doute qu'après la mort du connétable Anne en 1567, sa famille ne pesait plus autant qu'elle l'avait fait. Cela allait se sentir plus encore à partir de 1574, moment où François de Montmorency, son fils aîné, fut emprisonné, et où Henri, son second fils (Montmorency-Damville) gouverneur du Languedoc, assura solidement ses positions dans sa province, sans plus chercher à reconquérir à la Cour une influence trop fortement combattue par d'autres. Ni l'un ni l'autre, de toutes façons, n'avait à se soucier lui-même de la Bretagne, où la baronnie de Châteaubriant avait alors pour propriétaire Madeleine de Savoie, la veuve du connétable (jusqu'en 1586).

Ce fut l'arrivée de Jacques Barrin en Bretagne qui constitua la réponse à la demande qu'exprimait Myrevault en 1569. Elle nous confirme qu'on n'avait pas pu trouver dans la province de personnage assez influent et

32. Musée Condé, série L, t. XX, f ${ }^{\circ}$ 244, 23 février 1569, lettre de Myrevault à la duchesse de Montmorency. 
sûr pour s'y charger des intérêts des Montmorency, et elle nous montre que la connétable avait donc finalement choisi d'y envoyer un serviteur modeste, mais déjà bien connu et éprouvé, pour avoir été jusque là quotidiennement employé; et qu'elle avait choisi de lui faire acquérir des charges importantes au parlement et à la Chambre des comptes, pour lui donner plus de poids. Avec lui, nous avons un troisième type de serviteur de Montmorency à la Chambre des comptes : après les officiers de finances royaux les plus importants dans la province, qui venaient ajouter à leur charge le soin des affaires du duc, les deux responsabilités étant alors très voisines, et naturellement complémentaires; après les plus petits officiers qui avaient connu à l'origine le service (les recettes) d'un seigneur, mais qui cherchaient désormais à poursuivre leur carrière au service du roi, et cherchaient pour s'y aider la protection de quelque Grand, voici le cas d'un homme qui était d'abord et seulement un serviteur sûr, placé délibérément par la connétable elle-même à la Chambre des comptes dans l'intérêt de ses affaires (dont elle le chargeait explicitement par ailleurs), un peu comme Jean d'Alesso avait dû l'être trente ans plus tôt. Avec Barrin, le service de Montmorency n'était pas le moyen de circonstance d'aider à satisfaire une ambition personnelle, mais probablement l'objectif fondamental.

Jacques Barrin (env. 1540-1593), sieur des Roulliers, puis de la Haye, de la Galissonnière et du Boisgeffroy, n'était pas un Breton, mais était probablement né à Paris, d'une famille bourbonnaise. C'était un serviteur sûr des Montmorency, puisqu'il avait été élevé dans ce service, à l'ombre de son père ${ }^{33}$ et de son oncle Toussaint Barrin, sieur de Vincelles (qui présida plusieurs années le conseil parisien du duc). Il avait commencé très jeune à servir le connétable, comme l'un de ses secrétaires (" un petit clair et soliciteur ") assez pour être par lui pourvu d'abord de l'office de juge criminel à Angoulême (alors qu'il n'avait qu'une vingtaine d'années), puis (de 1564 à 1567) de celui de conseiller au parlement de Rennes ${ }^{34}$. C'est ce que rap-

33. Jacques Barrin était le fils de noble Pierre Barrin, sieur des Roulliers, qui était archer des gardes du corps du roi, à sa mort en 1557. Toussaint Barrin, sieur de Vincelles (mort en 1581), abbé de Saint-Romain de Blaye et abbé de Saint-Maurice, oncle de Jacques, aurait été officier dans la compagnie du connétable, avant de devenir prêtre et confesseur de la connétable, et de présider le conseil du duc. Jacques Barrin était sieur des Roulliers (terre du Bourbonnais, qu'il délaissa bientôt à son cadet), puis fut sieur de la Haye (en Derval), de la Galissonnière (en Saint-Jean de Béré), et enfin du Boisgeffroy (en Saint-Médard-sur-Ille).

34. Lettre de Montmorency au duc d'Étampes du 16 juillet 1564, Bibli. nat., ms. fr. 20510, $f^{\circ} 112$ : "Monsieur, j'ay faict pourvoir Barin, présent porteur, d'un estat et office de conseiller au parlement de Bretagne, où il s'en va pour être reçu, auquel j'ay recommandé vos affaires comme les myennes propres, et par luy n'ay voulu faillir vous faire la présente pour vous suplier, s'il a besoing de votre ayde et faveur envers vos amys audict Parlement pour sa réception, que vous l'en veuillez le couvrir pour l'amour de moy, affin que à ceste prochaine séance il puisse estre reçu. Je lui ai pareillement donné charge de vous dire de mes nouvelles et de celles de ceste compaignye qui s'en va à Rousilon... " Vingt-cinq ans plus tard, tout le monde à Rennes se souvenait encore des protections qui 
pelait en 1663 son petit-fils Jean Barrin du Boisgeffroy : " Nous devons à deffunt Monseigneur le connestable notre établissement en cette province $^{35}$. " Probablement s'intéressa-t-il déjà alors aux affaires de Châteaubriant, où il vint rejoindre le duc qui y séjournait en 1565, et où on l'avait d'ailleurs déjà vu auparavant, en novembre 1563 au moins, accompagnant une tournée d'inspection de l'intendant général de Montmorency (et sans doute y était-il déjà passé du temps du trésorier Charron). Il retourna à Paris après 1567, il y demeurait toujours en 1571.

Lorsque la connétable le renvoya en Bretagne, elle commença par le faire pourvoir, le 8 juin 1571, d'un office de président aux enquêtes au parlement de Rennes, et, deux ans après, le 13 février 1573, d'un office de président à la Chambre des comptes. Il put pendant quatre ans cumuler ces deux charges, par une faveur exceptionnelle qu'autorisèrent des lettres patentes royales : comme lors de son premier passage en 1564-1567, Barrin était bien venu en Bretagne en service commandé, poussé et appuyé par les Montmorency. Chacune de ses deux charges royales correspondait à un des aspects de sa fonction de superintendant des affaires de la duchesse dans la province, ce qu'il était en fait depuis 1571, avant même qu'un brevet du roi du 12 décembre 1572 le chargeât du " soin des affaires de Madame la connestable comme estant son intendant ". À Nantes, il n'était pas un officier des comptes entré dans la clientèle de la duchesse, mais un de ses serviteurs, pourvu d'un office important dans l'intérêt de ce service, lequel, en ce cas, rejoignait le sien propre, satisfaisait son ambition, on s'en doute, et amorçait la réussite familiale. La réussite de Barrin n'apparaît d'ailleurs pas seulement par les charges qu'il reçut, mais aussi par les biens qu'il acquit, et tous grâce à ses fonctions au service de son maître : la maison et métairie de la Haye, en Derval, achetée en 1576, était une partie du domaine de la baronnie de Châteaubriant; la seigneurie de la Galissonnière et les fiefs de la Rivière et de la Chauvelière (en Erbray), et de la Rivière-enHaut-Bois (en Saint-Julien de Vouvantes) en étaient mouvants, et situés aux portes du château. Quant à la seigneurie du Boisgeffroy (en Saint-Médardsur-Ille, au diocèse de Rennes), que Barrin acquit d'Albert de Gondi, maréchal de Retz en 1582, elle ne put lui revenir que parce qu'il avait décidé la connétable à vendre à Gondi sa terre de Callac. Ses fonctions d'intendant des domaines bretons des Montmorency lui ouvrirent donc des possibilités intéressantes, et orientèrent ses achats fonciers.

Cette réussite assez spectaculaire explique qu'il ait été considéré par ses ennemis comme un détestable parvenu, et accusé de tous les vices possibles (ainsi que cela se révéla par la suite, lors de la Ligue). En 1576 toutefois, il devint président à mortier au parlement, et il céda l'année suivante

avaient permis à Barrin d'entrer au Parlement : " [...] il apporta lettres de faveur de monsieur de Montmorency et autres Grands..." ("Dialogue de Maître Adrien Jacquelot... ", Bibli. nat., ms. fr. 22331).

35. Musée Condé, série P, t. XXVIII, 29 janvier 1663, lettre de Jean Barrin du Boisgeffroy au prince de Condé. 
son office à la Chambre des comptes. Sa charge et sa résidence rennaises l'éloignèrent dès lors de Nantes (comme elles l'empêchaient aussi de suivre ce qui se passait à Châteaubriant d'aussi près qu'il aurait fallu). On peut quand même penser que ce départ de Nantes ne fut pas, pour la duchesse, la fin de toute possibilité d'y faire défendre ses intérêts, car Barrin y avait pris femme, en épousant en 1573 Jeanne Ruiz, et, au moins par sa bellefamille, il conservait forcément quelques relations. Par ailleurs, Georges Godet, garde des livres en 1575, puis auditeur, et enfin maître des comptes en 1587, pouvait être utilisé, lui qui avait deux demi-frères au service de Montmorency : Julien Godet, sieur de la Villeharel, lieutenant, puis alloué de Châteaubriant; et Guillaume, sieur de Boon, procureur de la duchesse au parlement ${ }^{36}$. Il faut quand même rester prudent. Rien n'est venu nous prouver un engagement quelconque de Georges Godet au service des Montmorency. Il semble plutôt s'être surtout attaché à celui de la reine mère $^{37}$, comme le faisait au même moment la famille Meneust (nous l'avons dit plus haut), avec Gilles et Guy Meneust, frère et fils de l'auditeur des comptes, et probablement avec l'auditeur des comptes lui-même, Guillaume. La protection de Catherine de Médicis expliquerait l'anoblissement de Guillaume et de Guy (le père et le fils) le 29 décembre $1577^{38}$. La diminution de l'influence de Montmorency se devinerait à ce transfert de fidélité.

36. Noble homme Georges Godet, sieur de la Villeharel, ou sieur de la Touche (en Pléchâtel), baptisé à Saint-Sauveur de Rennes le 5 juillet 1550, était fils de Julien Godet, sieur de la Villeharel, et de Guillemette Guillemot (et petit-fils d'un autre Julien Godet). Il fut reçu garde des livres à la Chambre des comptes le 25 juin 1575, et résigna en 1582. Il fut reçu correcteur le 19 novembre 1583, puis maître des comptes le 6 juin 1587. Il mourut vers 1600. Julien Godet, son demi-frère, né en 1536, avait été nommé en février 1559 procureur du connétable au parlement de Rennes, et il fut ensuite pourvu, entre 1567 et 1570, de la charge de lieutenant de Châteaubriant. Guillaume Godet, sieur de Boon, son autre demi-frère (1542-1590), avocat au parlement, alloué de la juridiction de l'abbaye Saint-Georges de Rennes, fut procureur du comte de Laval aux États de Bretagne de 1580, et procureur des bourgeois de Rennes à ceux de 1585. Il était cité encore cette année-là comme avocat et conseil de madame la connétable au siège de Rennes, et on parlait aussi à cette date " des longs services que ses prédécesseurs et lui avaient faits au comte de Laval " (Arch. dép. d'Ille-et-Vilaine, 1 BA/ 8, f 227 v). On repère en effet un Julien Godet (le grand-père?) procureur de Tinténiac et châtelain de Montfort, pour le comte de Laval en 1521-1522, et, encore auparavant, en 1506, Maistre Julien Godet (le même personnage?), notaire et greffier de la juridiction de la vicomté de Rennes (qui appartenait elle aussi à la famille de Laval) (Arch. dép. d'Ille-et-Vilaine, $1 \mathrm{~F}$ 44-46). On pourrait même trouver des ancêtres plus reculés également serviteurs probables des Laval, en 1471, dans la châtellenie de Rougé-au-Theil. Charles Godet, sieur de la Villatte et du Tail, conseiller au parlement pourvu le 20 juin 1586, et mort en 1587 (que F. Saulnier identifie mal), était un cousin germain de ces trois frères et demi-frères Godet. Il était le fils de Maître Georges Godet, sieur du Tail, et de Roberde Even. Cette famille était très visible à Rennes au Xvi ${ }^{\mathrm{e}}$ siècle.

37. Le 20 février 1582, Georges Godet fut institué commis pour collecter en Bretagne les restes des sommes données à Catherine de Médicis pour financer les travaux du Louvre et des Tuileries. Il avait été choisi par Antoine Arnauld, sieur de la Mothe de Villeneuve, procureur général de la reine mère, Arch. dép. de Loire-Atlantique, B 61. f ${ }^{\circ}$ 154-156.

38. Arch. dép. d'Ille-et-Vilaine, 1 BA/7. f 304 v et 306 r. 
Si donc même Georges Godet, le seul officier des comptes que nous pourrions supposer lié à Montmorency dans les années 1580, ne l'était sans doute que de loin, on peut penser que le duc (ou la duchesse) n'avait pas grand monde à Nantes sur qui vraiment compter. À la fin du XVI ${ }^{\mathrm{e}}$ siècle, c'était plutôt de Rennes, où se trouvait Barrin (jusqu'à sa mort en 1593) qu'étaient surveillés les intérêts bretons de Montmorency. C'était au parlement, ou autour de lui, que se rencontraient surtout des serviteurs ou clients de la duchesse. Ce fut en tout cas là qu'il en réapparut peu à peu, vers la fin de la Ligue, lorsque la puissance retrouvée d'Henri I ${ }^{\mathrm{er}}$ de Montmorency auprès du roi, au fil des combats qu'ils menaient ensemble, suscita quelques sympathies intéressées. À Nantes, par contre, on manquait d'appui. Barrin, qui avait autre chose à faire que d'assurer le contrôle de la recette, auquel ne pouvait suffire le simple comptable présent à Châteaubriant, le donnait bien à penser en 1585 : Il est nécessaire « de nommer quelqu'un qui eût la charge de vos deniers casuels et des procès qui chaque jour s'intentent par vous pour la conservation de vos droits... Il est nécessaire de pourvoir de plus propre et habile à manier ce fait que votre greffier de Villemandi... Nous n'en trouvons point ${ }^{39}$..."

Le service de Montmorency, pendant la Ligue, put moins encore s'appuyer sur les officiers des comptes, même (semble-t-il) lorsqu'ils étaient fixés à Rennes. Du moins Roch Lezot, intendant de fait de la baronnie (après la mort de Barrin) ${ }^{40}$, suggérait-il que l'hostilité qu'il rencontrait (auprès d'eux entre autres, certainement) témoignait de leur mauvaise volonté à l'égard du duc lui-même :

" [...] Il y a beaucoup de personnes mal attentionnées au service du roy et de Monseigneur, entre aultres ceux de ce party qui sont pensionnés de Monsieur de Mercure. Monsieur le maréchal de Brissac le reconnaît bien et il n'y peut donner ordre... Il y a au Conseil de Monsieur le maréchal un dangereux homme qui est le sieur de la Touche Cornulier, qui a qualité de général et intendant des finances. Il m'a bien fait voir le peu d'attention qu'il a au service de Monseigneur et à la conservation de sa maison de Châteaubriant, écrivait-il le $1^{\text {er }}$ juillet $1597^{41}$."

39. Musée Condé, série L, tome XXI, f ${ }^{\circ}$ 328, 15 mars 1585, lettre de Barrin et Pierres (capitaine du château de Châteaubriant) à la duchesse de Montmorency.

40. Roch Lezot, sieur de Vaurozé, fils de Gilles, sieur de la Villegeffroy (greffier au présidial de Rennes dès 1556, secrétaire, puis contrôleur à la chancellerie de Bretagne) a été baptisé à Rennes (Saint-Germain) le 23 décembre 1567, et est devenu contrôleur en la chancellerie de Bretagne à la suite de son père, en 1589. Dès 1592, alors que Jacques Barrin vivait encore, il était au service du duc Henri I ${ }^{\text {er }}$ de Montmorency, puisqu'il s'occupait des affaires de Châteaubriant. En 1596, il devint receveur général de toutes les terres du duc en Bretagne et Anjou, et il le demeura jusqu'en 1609, mise à part une brève interruption en 1599-1601 lorsque fut tentée la ferme générale de ces terres.

41. Musée Condé, série L, t. XXXIV, f ${ }^{\circ} 4,1^{\text {er }}$ juillet 1597 , lettre de Roch Lezot au secrétaire Maridat. Claude de Cornulier, sieur des Croix, de la Haye et de la Touche, né à Nantes en 1568, devint trésorier de France et général des finances en 1588, en succédant à son père. Mercoeur l'enferma pendant dix mois au château de Nantes, et il fut ensuite commissaire du roi aux États de Rennes de 1590 et de 1593. Les Cornulier avaient grandi au service de la famille de Laval, dès le temps de Gui XIV (à la fin du XV siècle), époque à 
S'adressant au duc lui-même, le lendemain, il ajoutait que Cornulier était allé

" jusqu'à me dire que vous avez été cause de la guerre en cette province, ayant fait rompre la trêve par la prinse dudit Châteaubriant [...]. Aussi que le sieur de la Touche est créature de Monsieur le duc de Mercœur qui a encore à présent sa mère domestique de Madame de Mercœur, et son frère et beaufrère présidents à Nantes. Tous les siens y tiennent le premier rang ${ }^{42} \ldots$ "

"Il me promet d'employer tous ses amis afin d'offrir à Monsieur le duc de Mercœur la mauvaise vollonté qu'il me porte et les ombrages qu'il a de $\operatorname{moi}^{43} \ldots "$

Même prises avec quelque réserve, ces accusations de Lezot, tout en ne citant explicitement que Cornulier, n'encouragent pas à supposer à Montmorency de bien grands soutiens dans le milieu des officiers nantais. Claude de Cornulier, selon ces quelques mots, n'aurait pas montré d'attachement particulier au service du seigneur de Châteaubriant où son grand père, et ses ancêtres plus anciens, avaient pourtant été employés. Mais le lien solide ancien qui avait existé entre les Cornulier et les seigneurs de Châteaubriant s'était sans doute assez tôt dénoué, à la mort de Jean de Laval en 1542. Du temps des Montmorency, rien ne nous en est en tout cas apparu.

Par l'amenuisement de leur clientèle à la Chambre des comptes après 1567, et en tout cas dans le dernier quart du Xvi ${ }^{\mathrm{e}}$ siècle, on peut deviner le moindre pouvoir des Montmorency auprès du roi à cette période, par rapport aux années précédentes. Mais un infléchissement comparable se retrouvait pour les autres grands seigneurs bretons. Tous, comme Jean de Laval puis le connétable Anne, avaient eu avant 1550 une capacité de patronage efficace, qui tenait à ce que le roi (François ${ }^{\mathrm{er}}$ ) avait choisi, alors, de s'appuyer sur eux dans la province, d'autant plus, a-t-on remarqué, que ces Laval, Rieux et Rohan étaient par ailleurs ses cousins. Pour diverses raisons, dès le milieu du siècle, ils n'eurent plus assez de crédit auprès des derniers Valois pour qu'on cherchât à fonder sur eux autant d'espoirs de carrière qu'auparavant ${ }^{44}$. Le cas des ducs de Montmorency n'était donc pas un cas vraiment isolé.

laquelle ils seraient venus se fixer de la région de Vitré dans celle de Châteaubriant. Là, en tout cas, Pierre $1^{\text {er }}$ Cornulier, puis son fils Pierre II (mort en 1554) furent successivement capitaines des arquebusiers à cheval de François, puis de Jean de Laval. Pierre III Cornulier, lui (le père du général des finances du temps de la Ligue), se mit au service du duc d'Étampes, puis de Sébastien de Luxembourg, tous deux gouverneurs de Bretagne, comme leur secrétaire. Il fut aussi receveur des fouages, maître des comptes (en 1568), et trésorier général des finances en 1570.

42. Musée Condé, série L, t. XXXIV, f ${ }^{\circ}$ 14, 2 juillet 1597, lettre de Roch Lezot au duc de Montmorency.

43. Id., t. XXXVII, f ${ }^{\circ}$ 146, 24 octobre 1597, lettre de Roch Lezot au duc de Montmorency.

44. C'est ce que remarque Michel NASSIET, Parenté, Noblesse et États dynastiques, $X v^{e}$ et XVI siècles, Paris, éd. de l'EHEss, 2000. p. 306 : « Moins d'une génération plus tard (= après 1530), ce réseau royal de clients-cousins fut anéanti par les extinctions de lignées et par un phénomène qui allait bouleverser les données politiques, la division confessionnelle... 
Mais à partir de la Ligue non plus, nous ne parvenons plus du tout à trouver d'officiers des comptes qui pèsent réellement dans les affaires des ducs de Montmorency. Il n'y eut d'ailleurs même plus de vrai responsable breton de ces affaires, d'une envergure sociale comparable à celle du président Barrin dans les années 1570-1580, ou à celle de la Pommeraye et de Le Charron auparavant. À Paris, on se résigna à n'avoir comme interlocuteurs locaux que des personnages moins considérables : ainsi Roch Lezot, sieur de Vaurozé, homme de loi rennais, qui devint (sans le titre) intendant de la baronnie pendant les dix années qui suivirent la Ligue; ainsi bientôt après, et pour longtemps, Louis Chotard, un Angevin qui avait commencé par être le fermier général de la terre.

Sans doute les Parisiens qu'envoyait périodiquement le duc en inspection en Bretagne avaient-ils toujours besoin de faire des démarches à Nantes, et d'y suivre les affaires qui concernaient leur maître à la Chambre des comptes. Mais aucun des officiers qu'ils y rencontraient ne nous est apparu particulièrement attaché à son service. Jacques Barrin de la Galissonnière luimême, le fils du président Barrin d'avant la Ligue ${ }^{45}$, ne semble pas l'avoir été, pas plus au parlement, où il fut d'abord, qu'à Nantes, où il fut président aux comptes de 1616 à 1626. Lorsqu'en 1663 Jean Barrin du Boisgeffroy, conseiller au parlement et petit-fils du président Barrin, écrivit au Grand Condé pour rentrer dans sa clientèle et être chargé de ses affaires bretonnes, il voulut mieux fonder sa demande en rappelant les services que sa famille avait naguère rendus aux prédécesseurs (et à ceux) du Prince :

" Nous devons à deffunt Monseigneur le connestable nostre établissement dans cette province, l'accroissement de notre fortune à la bonté de Monseigneur le Prince votre père, et l'estime de notre famille à la protection de Votre Altesse ${ }^{46} \ldots$...

On remarque en cette phrase que, si Barrin mentionnait bien Anne de Montmorency et Henri II de Bourbon parmi les protecteurs de ses parents, il ne disait pas un mot des ducs Henri Ir et Henri II de Montmorency, dont ni son père (André Barrin du Boisgeffroy), ni son oncle (Jacques Barrin de la Galissonnière) n'avaient certainement cherché à se rapprocher. Lorsqu'il était à la Chambre des comptes, on le devine, Jacques Barrin de la

(par suite du protestantisme des Rohan et de l'extinction des lignes aînées des Laval et des Rieux, dont les biens passèrent au fils d'Andelot), le roi, au début des troubles, avait besoin de se constituer une noblesse seconde."

45. Jacques Barrin de la Galissonnière, (né vers 1578, mort à Paris en 1645) était le second fils du président Barrin d'avant la Ligue. Il fut conseiller au parlement de Bretagne de 1600 à 1610, puis fut pourvu d'une charge de maître des Requêtes, et fut président aux comptes de juin 1616 à 1626, date où il fut nommé conseiller d'État. Il fut commissaire du roi aux États de Bretagne en 1634. Dans cette carrière, il fut d'abord aidé par son mariage, en 1603, avec Vincente Ruellan, fille de Gilles Ruellan, sieur du Rocher-Portal, qui fut, depuis la Ligue jusqu'à sa mort en 1627, un financier omniprésent dans les affaires du roi en Bretagne, et qui était de la clientèle de Richelieu. François de Vignerot, sieur de Pontde-Courlay, neveu de Richelieu, devint aussi le neveu de Jacques Barrin lorsqu'il épousa en 1626 Françoise du Guémadeuc, petite-fille de Gilles Ruellan.

46. Musée Condé, série P, t. XXVIII, lettre du 29 janvier 1663. 
Galissonnière avait dû trouver d'autres moyens pour aider à ses affaires (probablement son beau-père le financier Gilles Ruellan) et faire s'intéresser à lui Richelieu. Que le prince de Condé l'ait connu à Paris, ce qui est vraisemblable, n'empêche pas que ce ne dut être pour lui qu'un appui secondaire (et tard venu). À la Chambre des comptes, de 1616 à 1626, Barrin de la Galissonnière avait sans doute peu à attendre des ducs de Montmorency, lesquels ne semblent pas, en effet, l'avoir utilisé ${ }^{47}$. Même à Rennes, d'ailleurs, une fois passées les premières années d'après la Ligue, peu de parlementaires étaient alors des clients sûrs. Il est bien probable que la moindre faveur d'Henri Ir ${ }^{\text {er }}$ de Montmorency auprès du roi (à partir de la découverte de la conspiration de Biron, en juin 1602) eut une part de responsabilité dans cet affaiblissement de sa clientèle bretonne, ainsi que ses séjours de plus en plus fréquents et prolongés dans son gouvernement du Languedoc. Mais il faut aussi incriminer la négligence du duc, son insouciance à susciter et entretenir les bonnes volontés qui s'offraient parfois, et qu'il ne savait pas cultiver.

Après sa mort, en 1614, ce comportement fut aussi celui de son fils le duc Henri II. Dans l'été 1626, lorsqu'il passa à Châteaubriant (voyage exceptionnel), puis à Nantes, il demanda pourtant au président aux comptes Louis de Harouys ${ }^{48}$, sieur de la Seilleraie, de prendre la direction de ses affaires bretonnes. Et nous savons qu'à l'automne Harouys séjourna deux jours à Châteaubriant pour les étudier avec les serviteurs présents sur place, et qu'en novembre encore il vint à Oudon, demander aux envoyés du duc, qui s'y trouvaient, un brevet « si on veut qu'il s'entremette des affaires $^{49}$ ». Mais le duc était alors déjà reparti de Paris en Languedoc, et les choses en restèrent là. Au printemps suivant, l'intendant Chotard semblait le

47. On aurait bien tort de croire que la famille Barrin entretint naturellement longtemps, à l'égard des Montmorency du XVII ${ }^{\mathrm{e}}$ siècle, une fidélité indéfectible, capable de modeler ses comportements et de lui dicter ses engagements. L'affirmer en 1663, ou le laisser entendre, pouvait à cette date avoir un certain poids (on sait en effet que le Grand Condé ne se montrait pas indifférent aux familles des anciens serviteurs de la maison de Montmorency, d'où il était issu par sa mère). Mais l'idée qu'un service familial ancien, même plus ou moins mis en sommeil, continuât d'imposer un sentiment de fidélité à travers les générations, créât des liens particuliers (et réciproques) éternels, et justifiât une bienveillance durable des seigneurs, cela, c'était alors une idée assez nouvelle. Autour

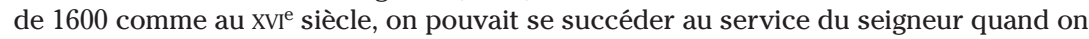
y faisait carrière, quand le père y introduisait son fils. On n'imaginait pas qu'un sentiment de fidélité familiale imprescriptible pût en découler, capable d'engager pour toujours les descendants des deux parties, et de leur valoir à jamais quelque traitement de faveur. $\mathrm{Au} \mathrm{XVIII}^{\mathrm{e}}$ siècle, au contraire, généralement, on affectait des deux côtés de trouver une telle idée justifiée, raisonnable, et honorable.

48. Louis de Harouys, sieur de la Seilleraie (1583-1656), président aux comptes en 1619, puis premier président en 1626 (jusqu'en 1632), par résignation de Jacques Barrin de la Galissonnière. Rien dans son ascendance, ni dans sa parenté, et pas même la personnalité de son prédécesseur en sa charge (qui ne s'en était pas mêlé, semble-t-il) ne le prédisposait à s'intéresser aux affaires de Montmorency.

49. Musée Condé, série L, t. CXII, f ${ }^{\circ}$ 53, 4 novembre 1626. Les deux envoyés du duc étaient MM. de Choisy et de Cornoaille. 
regretter : « Monsieur de Harouys... n'ayant reçu aulcunes nouvelles (des agents parisiens du duc), il estait demeuré les bras croisés ${ }^{50} \ldots$..

François Loysel, seigneur de Brie, président au parlement, qui avait été également pressenti, ne fut d'ailleurs pas non plus confirmé et entretenu dans ce genre de responsabilités : en aucune des deux grandes villes de la province, les deux derniers Montmorency ne surent se créer la clientèle que l'intérêt de leurs affaires aurait pourtant justifiée, et qui était pourtant à portée de la main. Mais le parlement de Rennes comptait quand même plus que la Chambre des comptes de Nantes, dont les membres sont totalement absents dans ce qui subsiste de la correspondance des ducs de Montmorency. Et nous le vérifions en notant qu'après 1632, lorsque le prince de Condé devint le nouveau possesseur de la baronnie de Châteaubriant, et qu'il se mit, lui, à entretenir une clientèle bretonne, il le fit seulement chez les parlementaires. Il ne devait connaître personnellement aucun des officiers des comptes, auxquels il lui arrivait pourtant d'écrire, car il demandait alors qu'on lui indiquât les noms de ceux qu'il fallait solliciter, ou qu'il fallait remercier pour leurs bons services. Il était beaucoup plus familier des sieurs de Quermagaro, de Coetlogon-Méjusseaume, de Bréquigny ou autres, qui étaient ses clients sûrs au parlement de Rennes. On ne peut citer comme un indice de relations de service ou de clientèle ce rappel que fit au Prince, en 1635, le sieur de la Roche Macé, d'un entretien qu'il avait eu peu avant avec lui, " m'ayant une fois en votre ville d'Oudon fait la faveur de me recepvoir à l'hommaige, de me parler de quelques affaires à traiter en la Chambre des comptes où je suis officier ${ }^{51}$... " Ce rappel venait à l'appui d'une sollicitation, et ne semble évoquer aucune relation constante entretenue, ni aucune familiarité avec le Prince.

$\mathrm{Au}$ XVII ${ }^{\mathrm{e}}$ siècle, donc, nous ne parvenons pas à identifier une clientèle qu'auraient eue les Montmorency à la Chambre des comptes, et nous avons même du mal à le faire pour leurs successeurs, qui se contentèrent d'utiliser les bonnes volontés occasionnelles qui s'y manifestaient, sans chercher à s'appuyer sur elles. Tout au plus le conseil du Prince de Condé acceptaitil à l'occasion, en considération de leurs charges d'officiers des comptes (qui pouvait présenter une utilité pour lui), d'accorder à certains une remise d'un tiers des droits dus pour tel achat de terre sous sa baronnie ${ }^{52}$. L'insuffisance des sources ne peut suffire à expliquer cette observation, dont il resterait à trouver les raisons. Elle peut d'ailleurs être faite aussi pour les comtes de Laval et barons de Vitré : alors qu'au Xvi ${ }^{\mathrm{e}}$ siècle ils avaient eu des

50. Id. série L, t. CXII, f ${ }^{\circ} 88,2$ avril 1627, lettre de Chotard au secrétaire Jacquelin.

51. Musée Condé, série M, t. V, f ${ }^{\circ} 37$, lettre du 28 février 1635. Jean Macé, sieur de la Roche, en Couffé, fut auditeur des comptes à la suite de son père Guillaume, de 1616 à 1645, et mourut en 1665 à Couffé, à 67 ans. Il avait épousé à Angers en 1620 Catherine Artault. Il était le beau-frère de René Chenu, sieur de Clermont, capitaine et gouverneur d'Oudon et Champtoceaux pour le prince de Condé.

52. Ainsi en mars 1719, pour une terre de 28000 livres acquise sous Vioreau par Jean Legrand de la Coustais, originaire de Châteaubriant et auditeur des comptes de 1715 à 1735 , musée Condé, 109 C 6 
serviteurs à la Chambre des comptes (Jean Apvril, sieur de Lourmais et son fils le sieur de la Grée, Antoine Dessefort peut-être auparavant...), il n'y eut plus rien de tel au XVII ${ }^{\mathrm{e}}$ siècle. Le parlement de Rennes fut alors la seule Cour où les ducs de la Trémoïlle eurent des clients importants issus de leur service (comme les Hay) ${ }^{53}$, ou favorables pour d'autres raisons, et que leur intendant Jean Grimaudet repérait bien comme tels en $1632^{54}$.

Vue de Châteaubriant, la Chambre des comptes ne fut dès lors que le point d'aboutissement des destinées familiales ascendantes de quelques dynasties de notables. Elle ne concerna plus guère la seigneurie qu'en ce qu'elle offrait des perspectives de carrière aux enfants de certains de ses fermiers et de ses juges. On quittait le service du seigneur pour poursuivre à Nantes la réussite familiale commencée à Châteaubriant, sans plus se sentir lié aux intérêts de son ancien maître, qui n'avait aucune part dans le choix de cette nouvelle carrière. Les exemples sont très nombreux de ces officiers des comptes dont les parents ou les grands parents avaient été mêlés aux recettes des seigneuries (comme d'autres aux fermes des impôts ou du domaine du roi). Mathurin Symon, sieur de Villeneuve, originaire de Nozay, auditeur des comptes de 1632 à 1656, avait pour grands parents paternels Julien Symon et Jeanne du Fresche, dont les deux familles avaient fourni des receveurs de Nozay aux XV et $\mathrm{XVI}^{\mathrm{e}}$ siècles $^{55}$. René Pactral, sieur de Monnol, correcteur des comptes en 1583, était lui aussi d'une famille de fermiers de Saffré ${ }^{56}$. Et il est bien probable qu'à Vitré, Mathurin Duverger, sieur de Boislebault, issu de lignées de fermiers de la baronnie au XVI ${ }^{\mathrm{e}}$ siècle, et lui-même sénéchal en 1619-1626, cherchait plus

53. Daniel Hay, sieur de la Motte et du Chastelet, lieutenant civil et criminel de Laval de 1591 à 1610, et intendant des affaires des comtes de Laval à partir de 1605, était le fils, le frère et le père de membres du parlement de Rennes. Il assurait lui-même, directement, aux comtes de Laval, un service qui se transmettait dans sa famille depuis des générations, et que ses parents parlementaires ne pouvaient avoir oublié. Son grand-père Jean Hay, sieur des Neptumières, procureur fiscal, puis (en 1527) sénéchal de Châtillon-enVendelais, était même pratiquement rentré dans la famille de Laval en épousant Claude Le Vairriez, fille de Jean Le Vairriez (Le Verryer), superintendant de Gui XVI, et d'Antoinette de Laval, sa " soeur naturelle".

54. " Ils ont tous obligation à votre maison, et croy aussi qu'il n'y en a aucun qui n'en ait du ressentiment ", écrivait Grimaudet au duc de la Tremoïlle le 27 décembre 1632 (Jean-Luc Tulot, Correspondance de Jean de Grimaudet, sieur de la Lande, intendant des La Tremoïlle à Vitré (1625-1651), Saint-Brieuc, 1999, dactyl.).

55. Mathurin Symon, sieur de Villeneuve, né à Nozay en 1605, auditeur des comptes reçu le 13 juillet 1632. Il existait aussi, dans la famille de sa mère, Guyonne Boulemer, et dans celle de sa femme, Gratienne Georgeteau, des précédents à sa carrière d'officier des comptes. À la fin du Xv siècle, Pierre Symon et Jehan du Fresche avaient été châtelains et receveurs de Nozay. Thomas Fresche avait été en 1535-1542 châtelain et fermier de Derval et Jans, et, après 1542, receveur de Vioreau. Jehan Fresche, sieur du Fouay, avait été fermier de Nozay en 1571, et Louis Fresche fermier de Derval vers 1580, et Jehan du Fresche, sieur du Perret, était intéressé dans les fermes de Nozay des années 1590.

56. Maître Pierre Pactral, sieur de Monnol, notaire royal en la Cour de Nantes, prit à ferme en 1520, pour trois ans, la terre et seigneurie de Saffré (marquis de l'Estourbeillon : "Inventaire des archives des châteaux bretons : Saffré "). Cette famille était au XVI siècle très familière des Avaugour, seigneurs de Saffré. 
à poursuivre une carrière personnelle, lorsqu'il devint maître des comptes en 1626, qu'à prolonger son service aux ducs de la Trémoïlle ${ }^{57}$. On voit de ces profils encore plus nettement aux débuts du XVIII ${ }^{\mathrm{e}}$ siècle, où confluèrent par exemple dans le milieu de la Chambre des comptes ces dynasties de fermiers et juges seigneuriaux qu'étaient les familles Urvoy (de Nozay), Davy (de Derval), Legrand et Le Normant (de Châteaubriant). Entre 1715 et 1721, quatre de leurs descendants furent presque simultanément reçus auditeurs des comptes : Jean Legrand, sieur de la Coustais (en 1715), et son frère Louis, sieur de la Lirais (en 1719), leur cousin Charles-François Le Normant de la Baguais, jusque là procureur fiscal de Châteaubriant (en 1721), et enfin Louis-Claude Hochedé de Belair (en 1719), héritier des Davy et Urvoy. On pourrait retrouver des promotions comparables dans l'histoire de quantité d'autres familles bretonnes, en d'autres terres, où on verrait également qu'aux générations des receveurs et des fermiers succédèrent la ou les générations des procureurs fiscaux, alloués et sénéchaux, puis celle des auditeurs des comptes (ou des conseillers au présidial), l'accès au parlement étant alors depuis longtemps largement hors de portée en ces milieux.

Les Montmorency, de 1542 à 1632, se préoccupèrent donc de moins en moins des officiers des comptes nantais. Les soutiens qu'ils y eurent au cours de ce siècle-là furent successivement de plusieurs types :

- celui des officiers des comptes (ou, plus largement, des officiers de finances) qui exerçaient à Nantes des responsabilités importantes, et y ajoutaient la conduite des affaires bretonnes du duc. Ces serviteurs-là, qu'Anne de Montmorency pouvait connaître de longue date, et avoir employés depuis longtemps, il n'y en eut plus après sa mort en 1567;

- celui des officiers des comptes moins importants, maîtres ou auditeurs, à qui la protection d'un Grand, quel qu'il fût, était utile pour soutenir une ambition de carrière. Nous en avons bien vu apparaître en 1571, qui n'étaient peut-être pas des cas isolés ou nouveaux. Mais ils n'eurent pas de successeurs pendant les trente années suivantes. Nous avons vu que les Meneust et Georges Godet reportèrent vers d'autres (la reine mère) leur recherche d'un protecteur;

- celui de Barrin, de 1573 à 1576, qui était d'abord un serviteur proche de Montmorency lorsqu'il vint en Bretagne (comme peut-être déjà Jean d'Alesso dès les années 1540), et qui le resta jusqu'à sa mort, ses charges royales à Nantes et à Rennes étant avant tout un moyen de mieux assurer le service de son maître. Elles lui permirent de fonder sa réussite familiale, et d'être tenu pour un parvenu exemplaire. Mais il n'eut pas non plus de successeur : ni son gendre Fouquet, ni ses fils André Barrin (au parlement)

57. Mathurin Duverger, sieur de Boislebault, sénéchal de Vitré de 1619 à 1626, maître des comptes de 1626 à 1633, fils de Mathurin Duverger, sieur du Pontdavy (1560-1632) et d'Olive Gérard. Il épousa en 1621 Olive Geffrard, elle aussi issue d'une des familles bourgeoises foisonnantes de Vitré. 
et Jacques Barrin (à la Chambre des comptes) ne semblent avoir joué un rôle dans les affaires du duc;

- et enfin celui des bonnes volontés occasionnelles, peut-être d'abord intéressées à leur propre réussite, peut-être aussi guidées par quelque tradition familiale, plus ou moins ancienne, de service au seigneur de Châteaubriant.

Après la Ligue, ce fut à ces bonnes volontés que se limitèrent les soutiens possibles de Montmorency à la Chambre des comptes, de même que ceux du prince de Condé après 1632. Elles ne permettent plus, à elles seules, de parler alors d'une véritable clientèle des ducs en ce milieu. Au contraire, $\mathrm{au} \mathrm{XVII}^{\mathrm{e}}$ siècle, des parlementaires rennais se présentaient parfois, et furent un moment utilisés, bien que toujours en deuxième ligne. On sent là comme la marque de la montée en puissance du parlement, et de la dévalorisation relative de la Chambre des comptes, et aussi comme la preuve que la protection d'un Grand n'avait plus comme naguère en Bretagne une importance décisive. Les officiers des comptes et parlementaires parisiens jouèrent plus longtemps un rôle déterminant dans le conseil et les affaires des ducs de Montmorency, et des princes de Condé après eux.

\section{RESUME}

Pour aider à la conduite de leurs affaires en Bretagne, où ils possédaient depuis 1542 la baronnie de Châteaubriant, les ducs de Montmorency disposaient comme d'autres de clients locaux. À la Chambre des comptes, cette clientèle, bien en vue, influente, dévouée et active au temps du connétable Anne de Montmorency (avant 1567), ne cessa ensuite de s'affaiblir, malgré l'envoi en cette Cour, dans les années 1570, d'un serviteur sûr, le président Jacques Barrin. Il n'eut pas de successeur. Au XVII ${ }^{\mathrm{e}}$ siècle, les Montmorency ne purent plus y compter que des sympathies occasionnelles et fragiles.

\section{ABSTRACT}

To manage their possessions in Brittany, where they owned the baronnie of Chateaubriant since 1542, the dukes of Montmorency had a network or relationships. At the Chambre des comptes, these conspicuous, influential and loyal friends, very active at the time of the constable Anne de Montmorency (before 1567), became weaker, in spite of the sending in this Court, in 1570, of a faithful servant, the president Jacques Barrin. It did not have a successor. During the XVII th century, the Montmorency had only occasional and unstable sympathies in this court. 
